\title{
Proteomic profiling for the identification of serum diagnostic biomarkers for abdominal and thoracic aortic aneurysms
}

\author{
Kazumi Satoh'1 , Tomoko Maniwa ${ }^{1}$, Teiji Oda ${ }^{2}$ and Ken-ichi Matsumoto ${ }^{1 *}$
}

\begin{abstract}
Background: Aortic aneurysm is an increasingly common vascular disorder with fatal implication. However, there is no established diagnosis other than that based on aneurysmal size. For this purpose, serum protein biomarkers for aortic aneurysms are valuable. Although most of the studies on serum biomarker discovery have been based on comparison of serum proteins from the patient group with those from the healthy group, we considered that comparison of serial protein profiles such as those in presurgical and postsurgical sera within one patient would facilitate identification of biomarkers since the variability of serial protein profiles within one patient is smaller than that between groups. In this study, we examined serum proteins with differential levels in postsurgery compared with those in presurgery after the removal of aneurysmal tissues in abdominal aortic aneurysm (AAA) and thoracic aortic aneurysm (TAA) patients in order to identify potential serum biomarkers for AAAs and TAAs.
\end{abstract}

Results: A proteomic approach with an isobaric tag for relative and absolute quantitation (iTRAQ) labeling followed by nano liquid chromatography (nanoLC)-matrix-assisted laser desorption ionization (MALDI)-time of flight (TOF/ TOF)-tandem mass spectrometry (MS/MS) was used. In the sera of patients with AAAs and TAAs, a total of 63 and 71 proteins with differential levels were further narrowed down to 6 and 8 increased proteins $(\geqq 1.3$ fold, postsurgical vs. presurgical) $(p<0.05$, patient vs. control) and 12 and 17 decreased proteins $(<0.77$ fold, postsurgical vs. presurgical) ( $p<0.05$, patient vs. control) in postsurgical sera compared with those in presurgical sera, respectively. All of the increased proteins in postsurgical sera of both AAA and TAA patients included several known acute-phase proteins. On the other hand, in the decreased proteins, we found intriguing molecules such as a-2-macroglobulin, gelsolin, kallistatin, and so on. Among them, we confirmed that kallistatin in both AAA and TAA patients and a-2-macroglobulin in TAA patients showed decrease levels in postsurgical sera similar to those in control sera by Western blot analysis with other sera from AAA and TAA patients.

Conclusions: Taken together, our findings suggest that Kallistatin and a-2-macroglobulin are potential serum biomarkers for both AAA and TAA and TAA, respectively.

Keywords: Abdominal Aortic Aneurysms, Differential Level Protein, iTRAQ, Serum Proteomics, Thoracic Aortic Aneurysms

\section{Background}

Aortic aneurysm is a severe cardiovascular disease with high mortality and morbidity. It is a complex disease with genetic and environmental risk factors. Aneurysms are described as abdominal aortic aneurysms (AAAs) and thoracic aortic aneurysms (TAAs) in terms of their

\footnotetext{
* Correspondence: matumoto@med.shimane-u.ac.jp

'Department of Biosignaling and Radioisotope Experiment, Interdisciplinary Center for Science Research, Organization for Research, Shimane University, Enya-cho, Izumo 693-8501, Japan

Full list of author information is available at the end of the article
}

anatomic location. It is well known that both AAAs and TAAs are associated with male gender, advanced age, cigarette smoking, hypertension, and chronic obstructive pulmonary disease. Both thoracic and abdominal aortas are large vascular conduits with similarities in their cellular components. However, thoracic and abdominal aortas are distinct in their biochemical and biomechanical properties, proteolytic profiles, inflammatory response, and genetic factors [1]. These differences are implicated in the distinct onset and expansion of aortic aneurysms in the thoracic and abdominal regions. A significant 
difference occurs in the distribution of aneurysms, and the most common location for aneurysms is in the infrarenal abdominal aorta, followed by the ascending thoracic aorta. In addition, it has been shown that intimal atherosclerosis, chronic transmural inflammation and elastic media destruction are associated with AAA growth, but many of TAAs arise in the absence of atherosclerotic plaque deposition. Preferably, TAAs are associated with the medial degeneration that is commonly related to the loss of vascular smooth muscle cells and destruction of medial elastic fibers [2].

Most patients with aortic aneurysms are asymptomatic until rupture of the aneurysm, which is often fatal. This disease is diagnosed when the diameter reaches $30 \mathrm{~mm}$ or more. However, there is no established medical therapeutics for small aneurysms. For this purpose, plasma/ serum protein biomarkers are available. Plasma or serum is considered a suitable body fluid for biomarker discovery since it is readily obtained via the least invasive mode without the risk of surgery. Studies are needed to find the serum/plasma biomarkers other than those for diagnosis based on aneurysmal size for the prediction of aneurysmal risk [3]. Proteome profiling of serum could also facilitate the discovery of AAA and/or TAA biomarkers for prognosis and therapeutic purposes for patients with aortic aneurysms. Circulating markers for the progression of AAA have been investigated [4,5]. These include extracellular matrix, matrix modulating proteases and their inhibitors, thrombogenic proteins, cytokines and inflammatory proteins, and lipids. Although a few of them have clinical potential for diagnosis, further confirmative and larger studies are needed. On the other hand, there were no reports about circulating biomarkers for TAA so far. It is conceivable that pathological disparities between AAA and TAA would bear different serum/plasma biomarkers related to each aneurysm.

The wide dynamic range of proteins and the presence of a few major proteins such as albumin and immunoglobulin complicate the analysis of serum/plasma proteomes. In addition, serum/plasma biomarkers would be factors released in very small amounts into the blood stream, making it difficult to detect them among other serum/plasma proteins that exist in much larger amounts. The removal of major proteins is a prerequisite for the identification of serum/plasma biomarkers in small amounts. This would increase the sample loading capacity and improve the efficiency of detection of proteins that exist in small amounts.

On the other hand, most of the studies on plasma/ serum biomarker discovery have been based on comparison of the mean values of plasma/serum proteins from the patient group with those from the healthy volunteer group. However, this approach sometimes gives rise to a large variation within each group, leading to masking of the variation that occurs between groups. Therefore, it is expected that the variability of serial protein profiles within one individual is smaller than that between groups [6]. Comparison of serial protein profiles within one individual would facilitate identification of biomarkers. We also expected that the postsurgical level of a certain protein would be restored to a level similar to that in normal control sera after resection of lesion parts. We anticipated that monitoring changes in serum proteomes that occur within each patient after surgical resection of aortic aneurysm tissues would readily provide biomarkers for clinical diagnosis and treatment and for understanding the molecular mechanisms underlying the development of AAA and TAA.

Recently, quantitative proteome analyses using tandem mass spectrometry (MS) with an iTRAQ labeling strategy have been developed and successfully applied to biomarker discovery for many conditions in both tissue [7] and serum samples [8]. In this study, serum proteins with differential levels in postsurgery compared with those in presurgery for the removal of aneurysmal tissues in AAA and TAA patients were investigated with iTRAQ labeling followed by nanoLC-MALDI-TOF/ TOF-MS/MS.

\section{Results}

Proteomic analyses of serum proteins with differential levels in postsurgical sera compared with those in presurgical sera of AAA and TAA patients

Presurgery and postsurgery serum samples were obtained from 7 AAA and 7 TAA patients who underwent aortic aneurysm resection. Protein levels in postsurgical sera were compared with those in presurgical sera using iTRAQ labeling coupled to nanoLC-MALDI-TOF/TOFMS/MS followed by ProteinPilot analysis. The average iTRAQ ratios of peptides in postsurgical sera to those in presurgical sera were calculated. A total of 180 differential level proteins in 7 AAA patients' sera and 199 proteins in 7 TAA patients' sera with 141 proteins detected in common in both groups were identified in at least one patient's serum (Figure 1A). Among those proteins, 78 differential level proteins in 7 AAA patients' sera and 86 proteins in 7 TAA patients' sera with 75 proteins detected in both groups were identified in at least 6 patients' sera in each group (Figure 1B). Relative quantitation by ProteinPilot analysis is based on statistical analysis. However, since most biochemical methods tend to have technical variation, we considered an additional cutoff value at 1.3 -fold change ( $\geqq 1.3$ fold or $<0.77$ fold, postsurgical $v s$. presurgical) for iTRAQ ratios for the selection of serum proteins with differential levels in postsurgical sera compared with those in presurgical sera [9-11]. Furthermore, among them, proteins in at least two samples in 4 volunteers' normal control sera for which iTRAQ ratios at the second time point (T2) to those at 
the first time point (T1) showed $\geqq 1.3$ fold or $<0.77$ fold (T2 vs. T1) were excluded. We used the "unused ProtScore" value $(>2)$ in ProteinPilot for an index of protein confidence (> 99\% confidence). Consequently, 63 proteins with differential levels in 7 AAA patients' sera and 71 proteins in 7 TAA patients' sera with 61 proteins detected in both groups were identified in postsurgical sera compared with those in presurgical sera with highly stringent criteria for protein identification (Figure 1C). Furthermore, the proteins that show statistical significant levels of postsurgical/presugical (patients' sera) compared with those of T2/T1 (control sera) $(p<0.05$, patient $v s$. control) were selected. Finally, 6 and 8 proteins with significantly increased levels ( $\geqq 1.3$ fold, postsurgical $v s$. presurgical) and 12 and 17 proteins with significantly decreased levels (< 0.77 fold, postsurgical vs. presurgical) were identified in AAA and TAA patients' sera, respectively (Figure 1D).

Increased ( $\geqq 1.3$ fold, postsurgical $v s$. presurgical) and decreased $(<0.77$ fold, postsurgical $v s$. presurgical) serum proteins in postsurgical sera compared with those in presurgical sera of AAA patients (Table 1) and TAA patients (Table 2) are listed in the order of iTRAQ ratios, respectively. Among them, we investigated the correlation between the ratios of proteins in postsurgical sera to those in presurgical sera with gender difference of patients that provided blood samples (Additional file 1). In increased proteins in postsurgical sera of AAA patients (Table 1), leucine-rich $\alpha$-2-glycoprotein (LRG1) was detected [male group (AAA patients \#AAA1, \#AAA2, \#AAA3, \#AAA4 and \#AAA5) vs. female group (\#AAA6 and \#AAA7), $p=0.0198$ (unpaired $t$-test)]. In increased proteins in postsurgical sera of TAA patients (Table 2), serum amyloid A protein was significantly detected [male group (TAA patients \#TAA1, \#TAA4, \#TAA5, and \#TAA6) vs. female group (\#TAA2, \#TAA3 and \#TAA7), $p=0.0046]$. In decreased proteins in postsurgical sera of TAA patients (Table 2), gelsolin (male vs. female, $p=0.0357$ ), histidinerich glycoprotein (male $v s$. female, $p=0.0132$ ), fibronectin (male $v s$. female, $p=0.0101$ ), $\alpha$-2-macroglobulin (male $v s$. female, $p=0.0198)$, and inter- $\alpha$-trypsin inhibitor heavy chain $\mathrm{H} 2$ (male $v s$. female, $p=0.0170$ ) were significantly
A

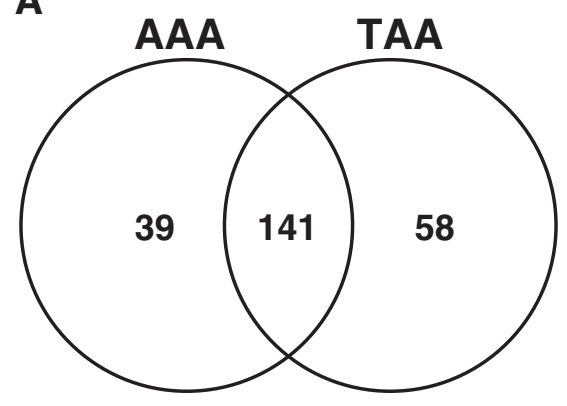

C

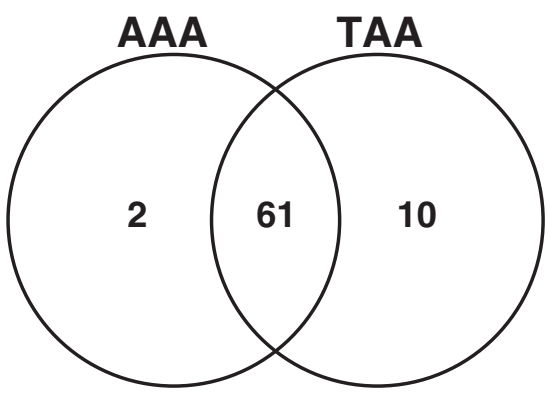

B

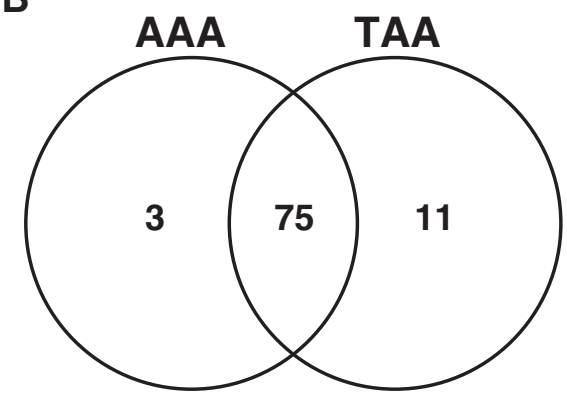

D

\begin{tabular}{|c|c|c|}
\hline \multirow{2}{*}{$\begin{array}{l}\text { iTRAQ ratio } \\
\text { (postsurgical vs. } \\
\text { presurgical) }\end{array}$} & \multicolumn{2}{|c|}{ Number of proteins } \\
\cline { 2 - 3 }$\geqq 1.3$ & 6 & 8 \\
\hline$<0.77$ & 12 & 17 \\
\hline
\end{tabular}

$(p<0.05$, patient vs. control)

Figure 1 Venn diagrams showing the number of proteins with differential levels in postsurgical sera compared with those in presurgical sera of patients with AAA and TAA. (A) Number of proteins that were identified in at least one sample among 7 samples in each group. (B) Number of proteins that were identified in at least 6 samples among 7 samples in each group. (C) Number of remaining proteins after exclusion of proteins for which ITRAQ ratios at the second time point (T2) to those at the first time point (T1) showed $\geqq 1.3$ fold or $<0.77$ fold in at least two samples in 4 control sera (T2 vs. T1). (D) Numbers of proteins with significantly increased $(\geqq 1.3$ fold, postsurgical vs. presurgical) and decreased level ratios (< 0.77 fold, postsurgical vs. presurgical) that were identified in at least 6 samples among 7 samples in sera of AAA and TAA patients and with statistical significant levels of iTRAQ ratio of postsurgical/presugical of patients' sera compared with that of T2/T1 volunteers' sera $(p<0.05$, patient vs. control). 
Table 1 Proteins with differential levels in postsurgical sera compared with those in presurgical sera of AAA patients

\begin{tabular}{|c|c|c|c|c|c|c|c|c|}
\hline Unused protScore ${ }^{\mathrm{a}}$ & $\%$ Coverage $^{\mathbf{b}}$ & Peptides` (95\%) & Uniplot number & Gene symbol & Protein name & $\begin{array}{c}\text { iTRAQ ratio }^{\mathrm{d}} \\
\text { average } \pm \mathrm{SE} \\
\text { (postsurgical vs. } \\
\text { presurgical) }\end{array}$ & $\begin{array}{l}\text { (patient vs. } \\
\text { control) }\end{array}$ & Molecular function \\
\hline \multicolumn{9}{|l|}{ Increased proteins } \\
\hline 37.7 & 86.9 & 28 & P02735 & SAA1 & Serum amyloid A protein & $8.09 \pm 3.60$ & $-{ }^{f}$ & Acute phase reactant \\
\hline 48.8 & 56.5 & 37 & P01011 & SERPINA3 & Alpha-1-antichymotrypsin & $1.88 \pm 0.12$ & 0.0006 & Protease inhibitor \\
\hline 53.1 & 67.7 & 56 & P02763 & ORM1 & Alpha-1-acid glycoprotein 1 & $1.85 \pm 0.21$ & 0.0250 & Transporter \\
\hline 26.2 & 54.5 & 14 & P02750 & LRG1 & Leucine-rich alpha-2-glycoprotein & $1.85 \pm 0.15$ & 0.0032 & Unknown \\
\hline 26.5 & 40.1 & 13 & P02748 & C9 & Complement component C9 & $1.36 \pm 0.09$ & 0.0135 & Complement \\
\hline 116.9 & 80.9 & 139 & P01009 & SERPINA1 & Alpha-1-antitrypsin & $1.32 \pm 0.06$ & 0.0081 & Protease inhibitor \\
\hline \multicolumn{9}{|l|}{ Decreased proteins } \\
\hline 26.1 & 30.4 & 12 & P06396 & GSN & Gelsolin & $0.55 \pm 0.03$ & 0.0000 & Actin scavenger \\
\hline 37.3 & 54.2 & 29 & P02765 & AHSG & Alpha-2-HS-glycoprotein & $0.61 \pm 0.05$ & 0.0002 & Extracellular matrix \\
\hline 18.5 & 74.0 & 17 & P02652 & APOA2 & Apolipoprotein A-II & $0.64 \pm 0.05$ & 0.0214 & Transporter \\
\hline 14.1 & 60.7 & 9 & P02753 & RBP4 & Retinol-binding protein 4 & $0.65 \pm 0.05$ & 0.0005 & Transfer/carrier protein \\
\hline 20.9 & 36.3 & 10 & P29622 & SERPINA4 & Kallistatin & $0.68 \pm 0.06$ & 0.0196 & Protease inhibitor \\
\hline 10.0 & 25.1 & 6 & P27169 & PON1 & Serum paraoxonase/arylesterase 1 & $0.69 \pm 0.06$ & 0.0010 & Hydrolase \\
\hline 89.6 & 89.9 & 110 & P02647 & APOA1 & Apolipoprotein A-I & $0.70 \pm 0.04$ & 0.0238 & Transporter \\
\hline 149.6 & 49.4 & 90 & P02751 & FN1 & Fibronectin & $0.72 \pm 0.06$ & 0.0330 & Extracellular matrix \\
\hline 163.9 & 82.5 & 176 & P02787 & TF & Serotransferrin & $0.73 \pm 0.04$ & 0.0004 & Transfer/carrier protein \\
\hline 36.9 & 50.3 & 20 & P04196 & HRG & Histidine-rich glycoprotein & $0.73 \pm 0.03$ & 0.0005 & Adapter protein \\
\hline 234.7 & 81.7 & 207 & P01023 & $\mathrm{A} 2 \mathrm{M}$ & Alpha-2-macroglobulin & $0.75 \pm 0.04$ & 0.0036 & Signaling molecule \\
\hline 44.3 & 35.7 & 26 & P19823 & $\mathrm{ITH} 2$ & $\begin{array}{l}\text { Inter-alpha-trypsin inhibitor heavy } \\
\text { chain } \mathrm{H} 2\end{array}$ & $0.77 \pm 0.06$ & 0.0024 & Protease inhibitor \\
\hline
\end{tabular}

${ }^{\mathrm{a}} \mathrm{A}$ score of protein confidence(ProtScore) for a detected protein that is calculated from the peptide confidence from spectra that are not already "used" by higher scoring proteins in the experiments. ${ }^{\mathrm{b}}$ The percentage of matching amino acids from identified peptides.

'The number of distinct peptides with at least $95 \%$ confidence in the experiments.

${ }^{\mathrm{i}}$ iTRAQ ratio of postsurgical sera compared with those in presurgical sera (postsurgical vs. presurgical).

'Statistical analysis of iTRAQ ratio of postsurgery/presurgery of patients' sera compared with that of T2/T1 of control volunteers' sera (patient vs. control).

fProtein that did not appear in control sera. 
Table 2 Proteins with differential levels in postsurgical sera compared with those in presurgical sera of TAA patients

\begin{tabular}{|c|c|c|c|c|c|c|c|c|}
\hline \multirow[t]{2}{*}{ Unused protScore $^{a}$} & \multirow[t]{2}{*}{$\%$ Coverage $^{\mathbf{b}}$} & \multirow[t]{2}{*}{ Peptides $^{c}$ (95\%) } & \multirow[t]{2}{*}{ Uniplot number } & \multirow[t]{2}{*}{ Gene symbol } & \multirow[t]{2}{*}{ Protein name } & \multirow{2}{*}{$\begin{array}{c}\text { iTRAQ ratio }^{\mathrm{d}} \\
\text { average } \pm \text { SE } \\
\text { (postsurgical vs. } \\
\text { presurgical) }\end{array}$} & \multirow{2}{*}{$\begin{array}{c}p \text { value }{ }^{\mathrm{e}} \\
\text { (patient vs. } \\
\text { control) }\end{array}$} & \multirow[t]{2}{*}{ Molecular function } \\
\hline & & & & & & & & \\
\hline \multicolumn{9}{|l|}{ Increased proteins } \\
\hline 46.2 & 82.0 & 48 & P02735 & SAA1 & Serum amyloid A protein & $15.65 \pm 3.40$ & $-{ }^{f}$ & Acute phase protein \\
\hline 16.0 & 34.8 & 9 & P02741 & CRP & C-reactive protein & $4.31 \pm 0.54$ & $-{ }^{f}$ & Acute phase protein \\
\hline 60.0 & 73.6 & 68 & P02763 & ORM1 & Alpha-1-acid glycoprotein 1 & $2.61 \pm 0.18$ & 0.0002 & Transporter \\
\hline 62.0 & 68.3 & 47 & P01011 & SERPINA3 & Alpha-1-antichymotrypsin & $2.39 \pm 0.21$ & 0.0010 & Protease inhibitor \\
\hline 29.8 & 59.1 & 24 & P02750 & LRG1 & Leucine-rich alpha-2-glycoprotein & $2.11 \pm 0.30$ & 0.0235 & Unknown \\
\hline 123.8 & 80.6 & 151 & P01009 & SERPINA1 & Alpha-1-antitrypsin & $1.50 \pm 0.15$ & 0.0397 & Protease inhibitor \\
\hline 28.3 & 32.7 & 16 & P02748 & C9 & Complement component C9 & $1.43 \pm 0.15$ & 0.0469 & Complement \\
\hline 16.5 & 21.7 & 8 & P09871 & C1S & Complement $\mathrm{C} 1 \mathrm{~s}$ subcomponent & $1.30 \pm 0.07$ & $f^{f}$ & Complement \\
\hline \multicolumn{9}{|l|}{ Decreased proteins } \\
\hline 30.2 & 38.0 & 15 & P06396 & GSN & Gelsolin & $0.49 \pm 0.05$ & 0.0002 & Actin scavenger \\
\hline 38.9 & 54.5 & 30 & P02765 & AHSG & Alpha-2-HS-glycoprotein & $0.51 \pm 0.09$ & 0.0008 & Extracellular matrix \\
\hline 19.3 & 89.0 & 14 & P02652 & APOA2 & Apolipoprotein A-II & $0.52 \pm 0.09$ & 0.0258 & Transporter \\
\hline 14.2 & 27.2 & 7 & P29622 & SERPINA4 & Kallistatin & $0.56 \pm 0.08$ & 0.0085 & Protease inhibitor \\
\hline 95.4 & 92.1 & 97 & P02647 & APOA1 & Apolipoprotein A-I & $0.59 \pm 0.09$ & 0.0473 & Transporter \\
\hline 16.5 & 53.7 & 10 & P02753 & RBP4 & Retinol-binding protein 4 & $0.60 \pm 0.10$ & 0.0027 & Transfer/carrier protein \\
\hline 183.4 & 83.7 & 171 & P02787 & TF & Serotransferrin & $0.60 \pm 0.08$ & 0.0021 & Transfer/carrier protein \\
\hline 23.1 & 67.6 & 11 & 095445 & APOM & Apolipoprotein M & $0.61 \pm 0.05$ & 0.0050 & Transporter \\
\hline 36.3 & 46.3 & 19 & P04196 & HRG & Histidine-rich glycoprotein & $0.61 \pm 0.07$ & 0.0061 & Adapter protein \\
\hline 156.2 & 49.9 & 98 & P02751 & FN1 & Fibronectin & $0.65 \pm 0.07$ & 0.0261 & Extracellular matrix \\
\hline 13.3 & 27.3 & 7 & P27169 & PON1 & Serum paraoxonase/arylesterase 1 & $0.66 \pm 0.07$ & 0.0011 & Hydrolase \\
\hline 16.4 & 22.3 & 7 & P03952 & KLKB1 & Plasma kallikrein & $0.66 \pm 0.09$ & 0.0191 & Protease \\
\hline 12.0 & 78.2 & 7 & P02655 & APOC2 & Apolipoprotein C-II & $0.66 \pm 0.14$ & 0.0387 & Transporter \\
\hline 289.1 & 78.8 & 228 & P01023 & $\mathrm{A} 2 \mathrm{M}$ & Alpha-2-macroglobulin & $0.66 \pm 0.05$ & 0.0027 & Signaling molecule \\
\hline 59.1 & 41.9 & 34 & P19823 & ITIH2 & $\begin{array}{l}\text { Inter-alpha-trypsin inhibitor heavy } \\
\text { chain } \mathrm{H} 2\end{array}$ & $0.68 \pm 0.08$ & 0.0043 & Protease inhibitor \\
\hline 28.4 & 38.4 & 14 & P43652 & AFM & Afamin & $0.70 \pm 0.08$ & 0.0041 & Transfer/carrier protein \\
\hline 30.5 & 44.7 & 18 & P05546 & SERPIND1 & Heparin cofactor 2 & $0.76 \pm 0.09$ & 0.0424 & Protease inhibitor \\
\hline
\end{tabular}

A score of protein confidence(ProtScore) for a detected protein that is calculated from the peptide confidence from spectra that are not already "used" by higher scoring proteins in the experiments.

${ }^{\mathrm{b}}$ The percentage of matching amino acids from identified peptides.

'The number of distinct peptides with at least $95 \%$ confidence in the experiments.

$\mathrm{d}_{\mathrm{i} T R A Q}$ ratio of postsurgical sera compared with those in presurgical sera (postsurgical vs. presurgical).

'Statistical analysis of iTRAO ratio of postsurgery/presurgery of patients' sera compared with that of T2/T1 of control volunteers' sera (patient vs. control).

fProtein that did not appear in control sera. 
detected. Furthermore we also examined the correlation between the ratios of proteins in postsurgical sera to those in presurgical sera with the number of days of blood sampling before and after surgery (Additional file 1). In increased proteins in postsurgical sera of AAA patients (Table 1), serum amyloid A protein was significantly detected [grouping based on the number of days before surgery, less than 1 week group (\#AAA1, \#AAA3 and \#AAA6) vs. more than 1 week group (\#AAA2, \#AAA4, \#AAA5 and \#AAA7), $p=0.0374$ ].

As mentioned below, among them two proteins (gelsolin and kallistatin) in patients' sera with AAA and three proteins (gelsolin, kallistatin and afamin) in patients' sera with TAA were finally examined by Western blot analyses with other presurgery and postsurgery blood samples. Furthermore, a positive acute-phase protein, $\alpha$-2-macroglobulin was also confirmed by Western blot analysis.

\section{Verification of iTRAQ ratio by Western blot analysis}

To confirm the accuracy of the quantitative results of iTRAQ ratios, some proteins were quantified again by Western blot analysis. The relative amounts of LRG1
(iTRAQ ratio $=1.99$, postsurgical $v s$. presurgical) and fibronectin (iTRAQ ratio $=0.69$, postsurgical $v s$. presurgical) for AAA patient \#AAA2 (Figures 2A,B,C,F) and LRG1 (iTRAQ ratio $=2.24$, postsurgical $v s$. presurgical) and fibronectin (iTRAQ ratio $=0.63$, postsurgical $v s$. presurgical) for TAA patient \#TAA4 (Figures 2A,B,D,G) in postsurgical sera compared with those in presurgical sera were examined by Western blot analysis. The ratios of levels of LRG1 and fibronectin in postsurgical sera compared with those in presurgical sera (1.0) in the AAA and TAA patients were determined by band intensity, and they were 1.62 -fold (Figure 2C) and 0.69-fold (Figure 2F), respectively, in sera of AAA patients and 1.37-fold (Figure 2D) and 0.63-fold (Figure 2G), respectively, in sera of TAA patients. Almost the same results were obtained in AAA patient \#AAA3 and TAA patient \#TAA1 (data not shown). Similarly, iTRAQ ratios of LRG1 (0.93) and fibronectin (0.96) in serum of a control volunteer \#C3 collected at two different time points ( $\mathrm{T} 1$ and $\mathrm{T} 2$ ) were almost identical to the ratios determined by Western blot analysis (LRG1, 1.01; fibronectin, 0.97) (Figures 2A,B,E,H). These results indicated that iTRAQ ratios are almost consistent with quantitative results obtained by Western blot analysis.

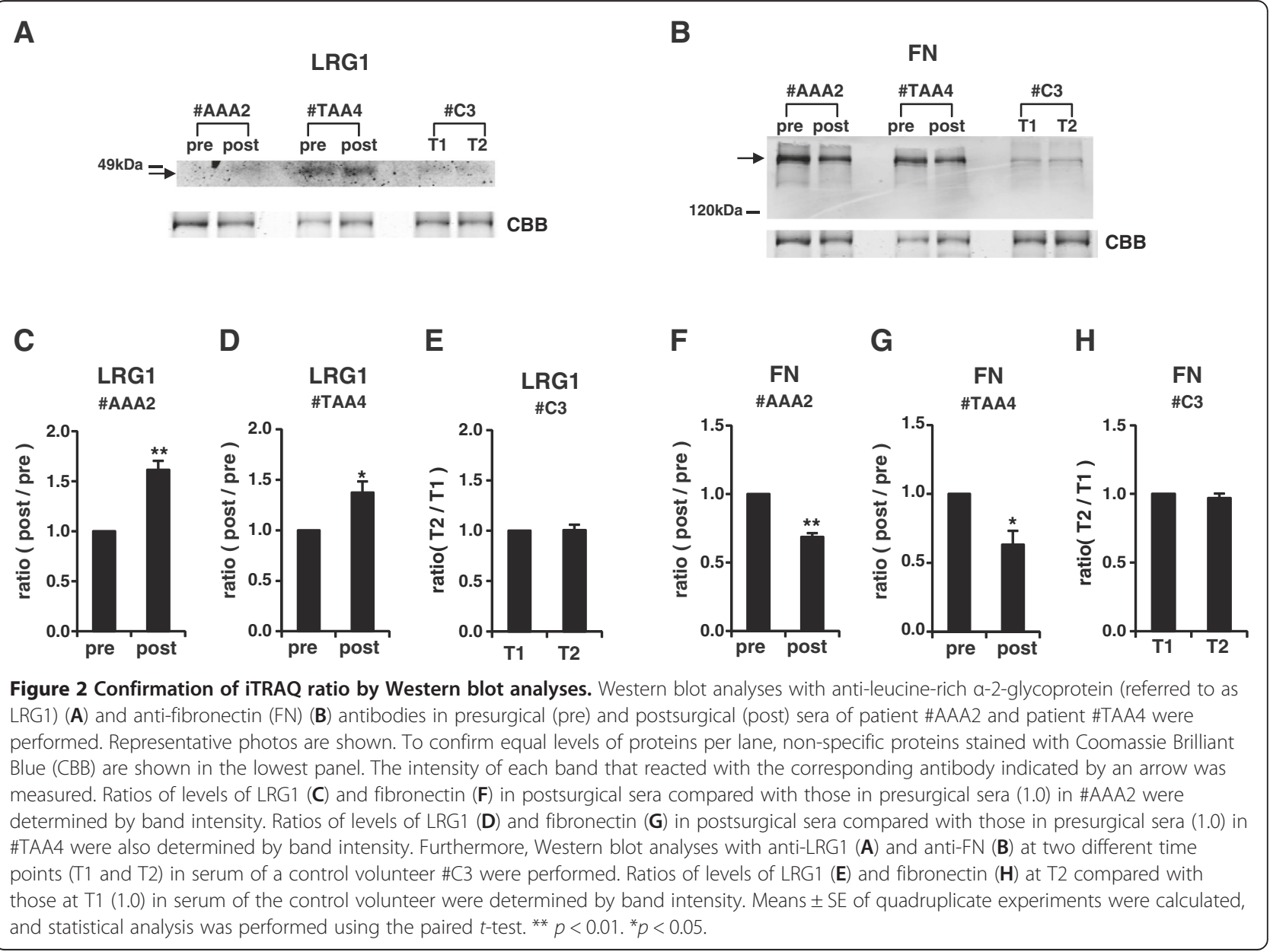




\section{Confirmation of differential levels of a-2-macroglobulin and kallistatin in presurgical and postsurgical sera by Western blot analysis}

All of the proteins on the list of serum proteins that were increased ( $\geqq 1.3$ fold, postsurgical $v s$. presurgical) in postsurgical sera compared with those in presurgical sera of AAA patients and TAA patients (Tables 1 and 2) were inflammatory response markers with increased levels during acute-phase response (see Discussion) [12-16]. These proteins include serum amyloid A protein, $\alpha-1$-antichymotrypsin, $\alpha$-1-acid glycoprotein 1 , LRG1, complement component C9, and $\alpha$-1-antitrypsin in both AAA and TAA patients (Tables 1 and 2). Creactive protein and complement $\mathrm{C} 1 \mathrm{~s}$ subcomponent were also increased in postsurgical sera of TAA patients (Table 2).

Intriguingly, five negative acute-phase proteins (retinolbinding protein 4 [17], fibronectin [18], serotransferrin [19], histidine-rich glycoprotein [20] and inter- $\alpha$-trypsin inhibitor heavy chain $\mathrm{H} 2$ [21]) were decreased in postsurgical sera compared with those in presurgical sera of both AAA and TAA patients. Some constituents of high- density lipoprotein (HDL) that are suppressed during the acute-phase response [22], apolipoprotein (apo) A-I, apo A-II, and serum paraoxonase/arylesterase 1 in the case of sera from AAA patients and apo A-I, apo A-II, apo C-II, apo $\mathrm{M}$, and serum paraoxonase/arylesterase 1 in the case of sera from TAA patients, were also included in the decreased proteins.

On the other hand, a positive acute-phase protein, $\alpha$ 2-macroglobulin, was unexpectedly decreased in postsurgical sera compared with that in presurgical sera of both AAA and TAA patients (Tables 1 and 2). Therefore, by Western blot analysis, we investigated the decreased levels of $\alpha$-2-macroglobulin, using postsurgical sera compared with presurgical sera from 12 other AAA patients and 10 other TAA patients, and examined whether the level of $\alpha$-2-macroglobulin in postsurgical sera is similar to that in normal control sera. If the level of $\alpha$-2-macroglobulin in postsurgical sera returns to a level similar to that in control sera, $\alpha$-2-macroglobulin would be a candidate of disease biomarkers for AAA and TAA. Consequently, we confirmed the decrease in $\alpha$-2-macroglobulin in postsurgical sera (Figure 3). In
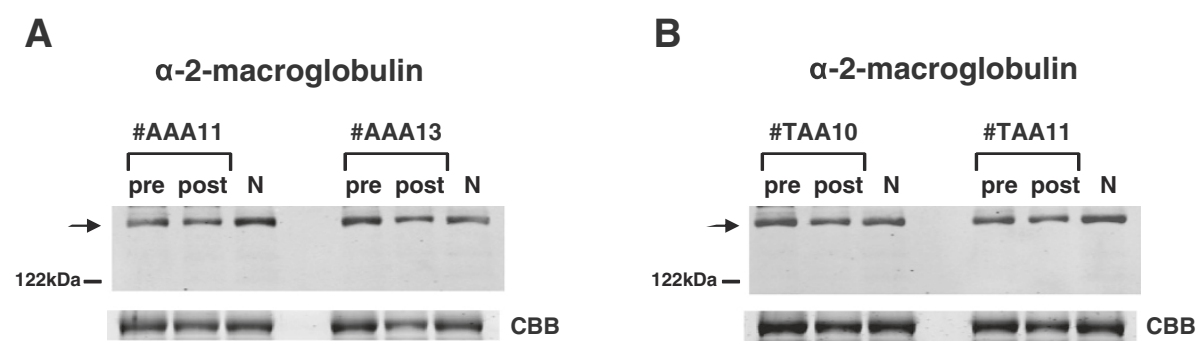

a-2-macroglobulin

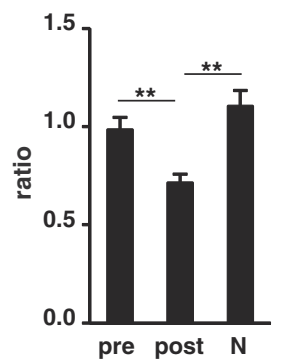

a-2-macroglobulin

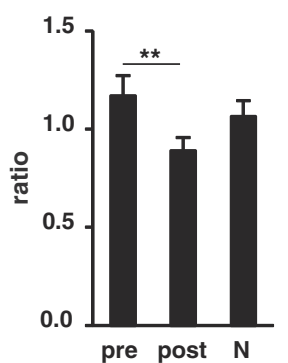

Figure 3 Examination of the decreased level of a-2-macroglobulin in postsurgical sera compared with that in presurgical sera of both AAA and TAA patients, and restoration of its level to that in normal control sera. Western blsot analyses with anti-a-2-macroglobulin antibody in presurgical (pre) and postsurgical (post) sera of 12 patients with AAA (A) and 10 patients with TAA (B) were performed as indicated by arrows. Representative photos of two each of patients (patient no. \#AAA11, \#AAA13, \#TAA10 and \#TAA11) as well as a mixture of 10 normal control sera $(\mathrm{N})$ are shown. To confirm equal levels of proteins per lane, non-specific proteins stained with Coomassie Brilliant Blue are shown (CBB). The intensity of each band in presurgical and postsurgical sera of 12 AAA and 10 TAA patients and 10 normal control sera that reacted with anti-a-2-macroglobulin antibody was measured. Ratios of levels of a-2-macroglobulin in presurgical and postsurgical sera and normal control sera compared with that in the mixture of 10 normal control sera (1.0) were determined by band intensity. Means \pm SE of triplicate experiments were calculated, and statistical analysis was performed using the paired $t$-test between presurgical and postsurgical data, whereas it was done using the unpaired $t$-test between postsurgical and normal control data. ${ }^{* *} p<0.01$. 
addition, the level of $\alpha$-2-macroglobulin in postsurgical sera of TAA patients (Figure $3 \mathrm{~B}$ ) but not in postsurgical sera of AAA patients (Figure 3A) was similar to that in normal control sera, indicating that $\alpha$-2-macroglobulin would be a candidate of disease biomarkers for TAA.

Among other decreased serum proteins in postsurgical sera compared with those in presurgical sera, we were interested in proteins such as gelsolin and kallistatin in AAA patients and gelsolin, kallistatin and afamin in TAA patients, for which detailed analyses in connection with aortic aneurysms have not been performed. Therefore, we further investigated these decreased levels of proteins by Western blot analysis (Figure 4). As a result, we confirmed decreased levels of proteins such as gelsolin and kallistatin in postsurgical sera of AAA patients (Figures 4A,B) and gelsolin, kallistatin, and afamin in postsurgical sera of TAA patients (Figures 4C-E) compared with those in presurgical sera by Western blot analysis. Subsequently, we investigated whether the protein levels in postsurgical sera are similar to those in normal control sera. In consequence, only the levels of kallistatin in postsurgical sera of both AAA and TAA patients were similar to those in normal control sera (Figures 4B,D). The level of gelsolin in the postsurgical sera of AAA patients (Figure 4A) and the levels of gelsolin and afamin in postsurgical sera of TAA patients (Figures 4C,E) were not similar to those in normal control sera. Thus, in postsurgical sera compared to presurgical sera only kallistatin showed a decreased level similar to that in normal control sera, indicating that kallistatin would also be a candidate of disease biomarkers for AAA and TAA.

\section{Discussion}

Serum proteins often possess information on diseases concerning the overall pathologic status of patients. Thus, circulating serum biomarkers could play an important role in the diagnosis of diseases and might also have a role in predicting progression of diseases. To identify critical molecular mechanisms underlying AAA formation, circulating serum biomarkers in patients and controls have been assessed in some case-control studies $[4,5]$. So far, most proteomic analyses attempting to reveal biological markers for AAA have been performed in AAA tissue samples [23]. Only one proteomic study for the identification of plasma biomarkers for AAA, which led to the identification of five differentially upregulated proteins in plasma samples of AAA patients compared to control patients, has been published [24]. However, the broad range of molecular alterations that occur in postsurgical sera compared to presurgical sera of one patient with AAA or TAA has not been elucidated. We speculated that a comparison of presurgical and postsurgical protein profiles within one patient would facilitate discovery of disease biomarkers. We expected that if the postsurgical level of a certain protein that shows a differential level between presurgical and postsurgical sera is restored to a level similar to that in normal control sera after the resection of AAA or TAA, the protein would be a disease biomarker. In this study, using an approach with iTRAQ labeling followed by nanoLC-MALDI-TOF/ TOF-MS/MS analysis, we determined proteomic profiles of proteins with differential levels in postsurgical sera compared with those in presurgical sera of AAA and TAA patients who underwent aortic aneurysm resections. Consequently, 6 and 8 proteins with increased level ( $\geqq 1.3$ fold, postsurgical vs. presurgical) and 12 and 17 proteins with decreased level ( $<0.77$ fold, postsurgical vs. presurgical) were identified in postsurgical sera compared with those in presurgical sera of AAA and TAA patients, respectively.

All of the proteins with increased levels ( $\geqq 1.3$ fold, postsurgical $v s$. presurgical) in postsurgical sera compared with those in presurgical sera of both AAA and TAA patients were positive acute-phase proteins such as serum amyloid A protein and $\alpha$-1-acid glycoprotein 1 . On the other hand, five serum proteins including retinol-binding protein 4, serotransferrin and others, and apolipoproteins such as apo A-I in HDL known as negative acute-phase proteins were decreased in postsurgical sera compared with those in presurgical sera of both AAA and TAA patients. Since postsurgical blood sampling was performed 4 to 20 days (Additional file 1 ) after surgical injury for aortic aneurysm resections, the increased levels of positive acute-phase proteins and the decreased levels of negative acute-phase proteins in postsurgical sera compared with those in presurgical sera might have been caused by the inflammatory response which is part of the normal response to surgical injury and not by specific resection of the diseased aortic aneurysmal tissues.

On the other hand, among other decreased proteins in postsurgical sera compared with those in presurgical sera, which we focused on and confirmed the decrease by Western blot analyses, we found that the decreased levels of kallistatin in both AAA and TAA patients' postsurgical sera and $\alpha-2$ -macroglobulin in TAA patients' postsurgical sera are restored to levels similar to those in normal control sera. These results indicate that kallistatin and $\alpha$-2-macroglobulin would be candidates of disease biomarkers for both AAA and TAA patients and TAA patients, respectively.

Kallistatin was initially discovered as a tissue kallikrein inhibitor and was shown to have multifaceted effects such as anti-inflammation, anti-oxidative stress, and antiangiogensis effects [25]. Administration of kallistatin prevented cardiac injury such as fibrosis and hypertrophy by inhibiting oxidative stress [26]. Since inflammation and oxidative-stress considerably arise in aortic aneurysmal tissues, the increase in kallistatin in patients' sera would be necessary to prevent cardiac remodeling and oxidative cardiac damage. 


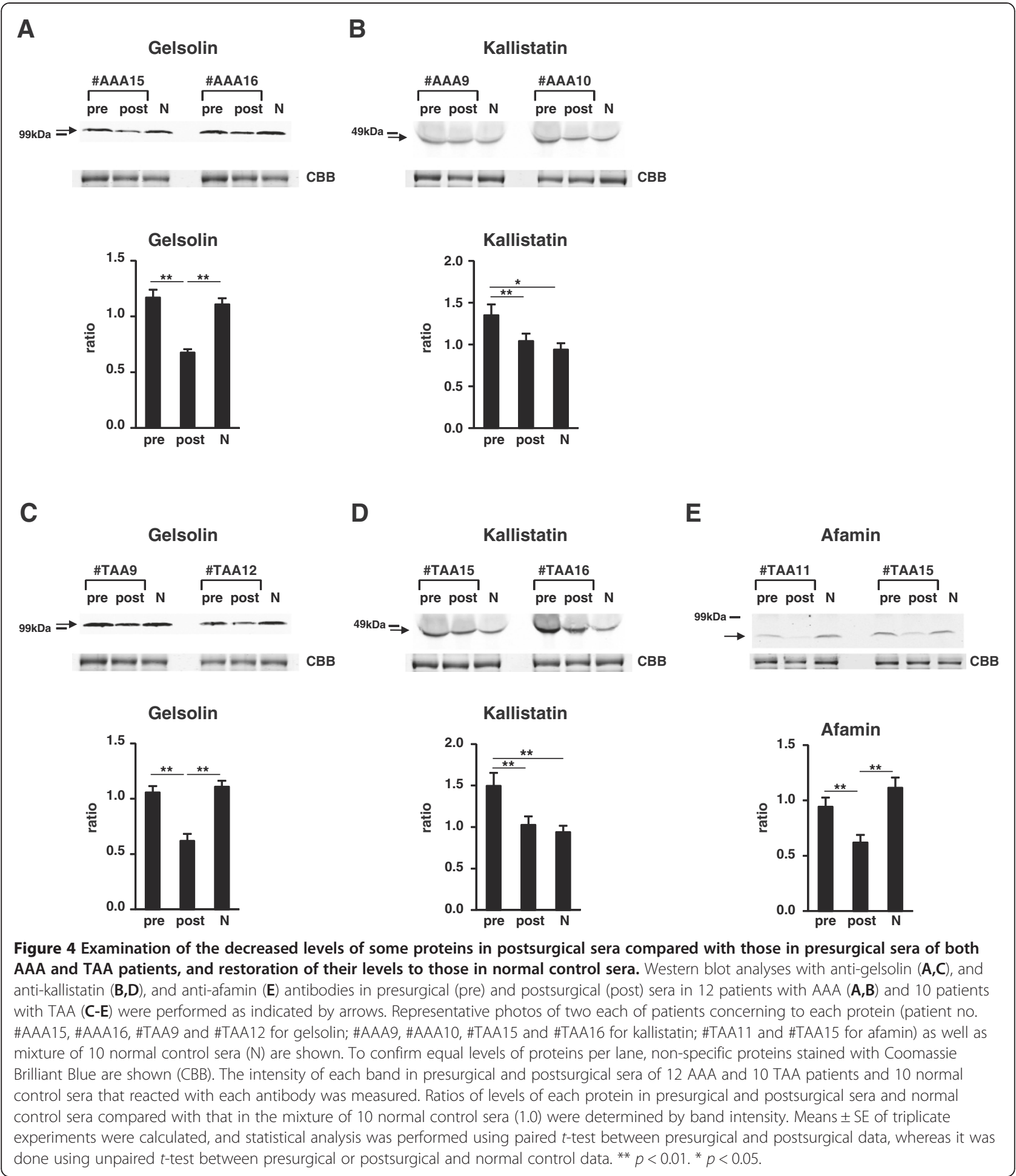

The primary function of $\alpha$-2-macroglobulin, one of the positive acute-phase proteins, is inhibition of a broad spectrum of proteases during tissue injury at sites of inflammation [27]. It has been reported that $\alpha$-2-macroglobulin is a valuable serum marker for the diagnosis of cardiac diseases [28]. Aortic aneurysms are characterized by inflammation with infiltration of inflammatory cells, induction of inflammatory cytokines, and release of reactive oxygen species. However, the differences in the physical structures of abdominal and thoracic aortas might result in differences in the inflammatory responses of AAA and TAA [2]. It is known that a Th2- 
predominant immune response plays an important role in AAA, whereas a Th1-predominant immune response occurs in TAA. These differences in inflammatory responses in AAA and TAA may contribute to the fact that $\alpha$-2-macroglobulin would be a candidate of disease biomarkers only in TAA patients.

Acosta-Martin et al. [24] identified 19 plasma diagnostic biomarker proteins potentially involved in AAA biological mechanisms using quantitative MS analysis. Among them, five proteins such as kallistatin, protein AMBP, gelsolin, heparin cofactor 2, $\alpha$-2-antiplasmin were found in common in the 63 proteins disclosed in AAA patients' sera in the present study. Moreover, they verified the increased levels of kallistatin and protein AMBP in individual plasma of AAA patients by Western blot analysis. In agreement with this, in the present study the level of kallistatin in presurgical sera was higher than in postsurgical sera and control sera as shown in Table 1 and Figure 4B. Furthermore, in the present study the level of protein AMBP in presurgical sera was moderately higher than that in postsurgical sera (iTRAQ ratio $=1.17$, presurgical $v s$. postsurgical). These evidences more strengthen the idea that kallistatin is a candidate of disease biomarkers for AAA.

So far some extracellular matrices such as aminoterminal propeptide of type III procollagen (PIIINP) and carboxyterminal propeptide of type I procollagen (PICP), thrombus-associated proteins such as fibrinogen, D-dimer and tissue plasminogen activator (tPA) and matrixdegrading enzymes such as matrix metalloproteinase-9 (MMP-9) have been reported as potential circulating biomarkers for AAA diagnosis in case-control study [4]. Interestingly, SanGiorgi et al. [29] showed that MMP-3 and MMP-9 are effective monitors from 1 to 6 months after AAA surgery. However, these known circulating biomarkers associated with AAA could not be identified in the present study. The levels of these circulating biomarkers in AAA patients might remain high for a while after the surgery, and they might not change in the investigated periods ( 4 to 12 days) after the surgery compared with those in presurgery.

\section{Conclusions}

Proteomic analyses of postsurgical sera compared with presurgical sera of patients with AAA and TAA were performed by using iTRAQ labeling followed by nanoLCMALDI-TOF/TOF-MS/MS analysis. Among identified proteins with differential levels between postsurgical sera and presurgical sera, kallistatin in both AAA and TAA patients' sera and $\alpha$-2-macroglobulin in TAA patients' sera might be candidates of serum biomarkers for aortic aneurysms. Our results provide valuable information on the underlining mechanisms of AAA and TAA and will contribute to the establishment of a method for early diagnosis of aortic aneurysm and to the development of pharmacological therapies.

\section{Methods}

\section{Human serum samples}

Blood samples were collected after approval from the Ethics Committee of Shimane University School of Medicine, Japan. The participants gave informed consent. Blood samples for quantitative proteome analyses were collected at presurgery and postsurgery from 7 AAA patients (5 males, 2 females; average age, 73.9 years) and 7 TAA patients ( 4 males, 3 females; average age, 73.1 years) who underwent aortic aneurysm resection during approximately 2 years in Shimane University Hospital. Presurgical and postsurgical blood samples were taken at an average of 7.1 days before and an average of 9.3 days after surgery, respectively. Atherosclerosis with calcification was macroscopically visible in most of the aneurysmal specimens from each patient. As normal control samples for quantitative proteome analyses, blood samples were collected from 4 healthy volunteers ( 2 males, 2 females; average age, 42.0 years) at two time points [first time point (T1) and second time point (T2)] with a 14-day interval. Age, gender, and time of sample collection of patients and control volunteers were shown in Additional file 1. Other presurgery and postsurgery blood samples were collected from patients with AAA ( 8 males, 4 females; average age, 79.4 years) and TAA (7 males, 3 females; average age, 67.6 years) who underwent aortic aneurysm resection during approximately 2 years in Shimane University Hospital for confirmation by Western blot analysis. Mixture of sera collected from 10 healthy volunteers ( 7 males, 3 females; average age, 42.0 years) was used as normal control samples for Western blot analysis. Age, gender, and time of sample collection of other patients and other control volunteers were shown in Additional file 2. Age matching of patients and volunteers was not fulfilled in the present study. Blood samples were centrifuged and the plasma layer was removed. Serum samples were stored at $-80^{\circ} \mathrm{C}$ until use.

\section{Immunodepletion of abundant serum proteins}

To remove the two most-abundant serum proteins [albumin and immunoglobulin (Ig)G], an immunodepletion column (Albumin \& IgG depletion SpinTrap) from GE Healthcare (Buckinghamshire, UK) was used according to the manufacturer's instructions. Briefly, the column was equilibrated with binding buffer $(20 \mathrm{mM}$ sodium phosphate, $0.15 \mathrm{M}$ sodium chloride). After centrifugation, $50 \mu \mathrm{l}$ of serum was diluted with an equivalent volume of binding buffer and then applied to the column and incubated for $5 \mathrm{~min}$. After centrifugation for $30 \mathrm{~s}$ at $800 \mathrm{~g}$, the eluate was collected. A further $100 \mu \mathrm{l}$ of binding buffer was applied to the column and centrifuged. This elution 
step was performed again. The eluate was combined and then desalted, and its buffer was exchanged with $50 \mathrm{mM}$ triethylammonium bicarbonate (TEAB) (Sigma, Tokyo, Japan) using spin concentrators (Corning, Tokyo, Japan). The protein concentration was determined using a bicinchoninic acid (BCA) Protein Assay Reagent (Thermo Fisher Scientific, Rockford, IL, USA).

\section{Denaturation, reduction, trypsin digestion, iTRAQ labeling, and strong cation exchange (SCX) chromatography}

Sample preparation was performed accordingly to the manual supplied by AB Sciex (Foster, CA, USA) and our previous paper [30]. Briefly, equal amounts of immunodepleted presurgical and postsurgical serum samples were denatured by sodium dodecyl sulfate (SDS) and reduced by [tris(2-carboxyethyl)phosphine (TCEP)]. Then cysteine alkylation was performed with methylmethanethiosulfonate (MMTS). Thereafter, each sample was digested by trypsin (AB Sciex). Each digest was labeled with a different iTRAQ tag by an iTRAQ Reagent Multiplex kit (AB Sciex). iTRAQ label 114 or 115 was used for labeling presurgical serum samples, and iTRAQ label 116 or 117 was used for postsurgical serum samples. Then the labeled presurgical and postsurgical samples were combined. This experiment was conducted for each patient. For the control samples collected at two time points, the former sample was labeled with iTRAQ label 114 or 115 and the latter sample was labeled with iTRAQ label 116 or 117 . Then the labeled samples were combined. This experiment was conducted for each volunteer. After that, the combined samples were fractionated into six fractions by strong cation exchange (SCX) chromatography according to the instructions of the manufacturer (AB Sciex). Then each fraction was desalted by a Sep-Pac $\mathrm{C}_{18}$ cartridge according to the instructions of the manufacturer (Waters, Milford, MA, USA).

\section{NanoLC and MALDI-TOF/TOF MS/MS analysis}

One fraction from SCX chromatography was fractionated to 171 spots with a DiNa nanoLC system (KYA Tech, Tokyo, Japan) and collected onto an Opti-TOF LC/MALDI 384 target plate (AB Sciex) using a Dina MaP fraction collector (KYA Tech) according to the instructions of the manufacturer (KYA Tech) and our previous paper [31]. Offline spotted peptide samples were analyzed on a 5800 MALDI-TOF/TOF MS/MS Analyzer with TOF/TOF Series software (version 4.0) (AB Sciex). MS spectra were acquired between $m / z 800$ and 4000 with positive ion mode. Parent ions of des-Arg1-bradykinin, angiotensin I, Glu1fibrinopeptide $\mathrm{B}$, adrenocorticotrophic hormone (ACTH) (clip 1-17), ACTH (clip 18-39) and ACTH (clip 7-38), diluted in a matrix $[4 \mathrm{mg} / \mathrm{ml} \alpha$-cyano-4-hydroxycinnamic acid (CHCA), Wako, Osaka, Japan], were used for calibration. Monoisotopic precursor selection for MS/MS was carried out by automatic precursor selection with an interpretation method using the DynamicExit Algorithm (AB Sciex). MS/MS data obtained by the 5800 MALDITOF/TOF were analyzed by ProteinPilot ${ }^{\mathrm{Tm}}$ software (version 3.0) with the Paragon protein database search algorithm (AB Sciex) [32]. Statistic method of iTRAQ analysis was according to ProteinPilot ${ }^{\mathrm{Tm}}$ software (version 3.0). Each MS/MS spectrum was searched against the database constructed by AB Sciex (version 20081216, 20,489 entries). Based on the iTRAQ ratio of postsurgery to presurgery of each peptide, quatitative changes of each protein in presurgical and postsurgical sera were calculated. The comparison between the iTRAQ ratio of postsurgery to presurgery of patients and that of T2 to T1 of control volunteers was statistically performed by the unpaired $t$-test (two side test), with $p<0.05$ being considered statistically significant.

\section{Bioinformatic analysis}

Panther software (version 7.2) (http://www.pantherdb. org/) was used for protein classification analysis. The annotations of identified proteins were acquired from the Uniprot database (http://www.uniprot.org/) and appropriate literature.

\section{Western blot analysis}

Crude sera for the analyses of $\alpha$-2-macroglobulin, kallistatin, gelsolin and afamin or albumin- and IgG-depleted sera for the analyses of fibronectin and leucine-rich $\alpha$-2-glycoprotein (LRG1) were electrophoresed through sodium dodecyl sulfate-polyacrylamide gel (SDS-PAGE), and then the proteins were transferred onto Hybond ECL nitrocellulose membranes (GE Healthcare Japan, Hino, Japan). The amount of crude sera $(\mu \mathrm{l})$ and albumin- and IgG-depleted sera $(\mu \mathrm{g})$ used for each analysis was as follows: $\alpha$-2-macroglobulin $(0.5 \mu \mathrm{l})$, kallistatin $(2 \mu \mathrm{l})$, gelsolin $(0.5 \mu \mathrm{l})$, afamin $(2 \mu \mathrm{l})$, fibronectin $(2.5 \mu \mathrm{g})$ and LRG1 $(3.3 \mu \mathrm{g})$. Western blot analyses were performed as described in our previous paper [33]. The membranes were reacted with rabbit polyclonal anti-fibronectin antibody (Sigma-Aldrich, St. Louis, MO, USA), mouse monoclonal anti-LRG1 antibody (Abnova, Taipei, Taiwan), rabbit polyclonal anti- $\alpha$-2-macroglobulin antibody (Abcam, Tokyo, Japan), goat polyclonal anti-kallistatin antibody (R \& D Systems, Minneapolis, MN, USA), mouse monoclonal anti-gelsolin antibody (SigmaAldrich, St. Louis, MO, USA) or goat polyclonal antiafamin antibody (GeneTex, Irvine, CA, USA). Then the proteins on the nitrocellulose membranes were reacted with anti-rabbit, anti-mouse or anti-goat Alex Flour 680-conjugated or IRDye 800-conjugated IgG (LI-COR, Lincoln, NE, USA), followed by visualization using the infrared imaging system Odyssey (LI-COR). The intensity of each band reacted with a corresponding antibody was measured for densitometric analyses of each protein level. Data 
from at least triplicate experiments were analyzed for statistical significance by the $t$-test (two side test). Its significance was set with $p<0.05$. Results are expressed as means \pm standard error (SE).

\section{Additional files}

Additional file 1: Sera used for iTRAQ labeling followed by nanoLCMALDI-TOF/TOF-MS/MS analysis.

Additional file 2: Sera of other patients used for Western blot analysis.

\section{Abbreviations}

AAA: Abdominal aortic aneurysm; TAA: Thoracic aortic aneurysm; ITRAQ: Isobaric tag for relative and absolute quantitation; nanoLC: Nano liquid chromatography; MALDI: Matrix-assisted laser desorption ionization; TOF: Time of flight; MS: Mass spectrometry; MS/MS: Tandem mass spectrometry; LRG1: Leucine-rich a-2-glycoprotein; FN: Fibronectin; CBB: Coomassie brilliant blue; HDL: High-density lipoprotein; Apo: Apolipoprotein; Ig: Immunoglobulin; TEAB: Triethylammonium bicarbonate; BCA: Bicinchoninic acid; SCX: Strong cation exchange; SDS: Sodium dodecyl sulfate; TCEP: Tris-(2-carboxyethyl)phosphine; MMTS: Methylmethanethiosulfonate; ACTH: Adrenocorticotrophic hormone; CHCA: a-cyano-4-hydroxycinnamic acid; SDS-PAGE: Sodium dodecyl sulfate-polyacrylamide gel; SE: Standard error.

\section{Competing interests}

The authors declare that they have no competing interests.

\section{Authors' contributions}

KS performed the sample preparation, peptide labeling and separation, MS analysis, raw data processing and analysis, bioinformatics, Western blot experiments, and statistic analysis. TM performed data analysis and bioinformatics. TO carried out sample collection and data interpretation. KM carried out the conception and design, data analysis and interpretation, and manuscript writing. All authors read and approved the final manuscript.

\section{Acknowledgements}

We thank Yasuko Sonoyama for sample collections. This work was supported in part by Grants-in-Aid for Scientific Research (22590063 to K.M.) from the Ministry of Education, Culture, Sports, Science and Technology of Japan and a research grant (to K.M.) from Daiwa Securities Health Foundation.

\section{Author details}

'Department of Biosignaling and Radioisotope Experiment, Interdisciplinary Center for Science Research, Organization for Research, Shimane University, Enya-cho, Izumo 693-8501, Japan. ${ }^{2}$ Division of Cardiovascular and Thoracic Surgery, Department of Surgery, Faculty of Medicine, Shimane University, Izumo, Japan.

Received: 12 December 2012 Accepted: 21 June 2013

Published: 27 June 2013

\section{References}

1. Ruddy JM, Jones JA, Spinale FG, Ikonomidis JS: Regional heterogeneity within the aorta: relevance to aneurysm disease. J Thorac Cardiovasc Surg 2008, 136:1123-1130.

2. Guo DC, Papke CL, He R, Milewicz DM: Pathogenesis of thoracic and abdominal aortic aneurysms. Ann NY Acad Sci 2006, 1085:339-352.

3. Sakalihasan N, Limet R, Defawe OD: Abdominal aortic aneurysm. Lancet 2005, 365:1577-1589.

4. Golledge J, Tsao PS, Dalman RL, Norman PE: Circulating markers of abdominal aortic aneurysm presence and progression. Circulation 2008, 118:2382-2392.

5. Urbonavicius S, Urbonaviciene G, Honoré B, Henneberg EW, Vorum $\mathrm{H}_{4}$ Lindholt JS: Potential circulating biomarkers for abdominal aortic aneurysm expansion and rupture-a systematic review. Eur J Vasc Endovasc Surg 2008, 36:273-280.
6. Roelofsen H, Alvarez-Llamas G, Dijkstra M, Breitling R, Havenga K, Bijzet J, Zandbergen W, de Vries MP, Ploeg RJ, Vonk RJ: Analyses of intricate kinetics of the serum proteome during and after colon surgery by protein expression time series. Proteomics 2007, 7:3219-3228.

7. Sutton CW, Rustogi N, Gurkan C, Scally A, Loizidou MA, Hadjisavvas A, Kyriacou K: Quantitative proteomic profiling of matched normal and tumor breast tissues. J Proteome Res 2010, 9:3891-3902.

8. Bijian K, Mlynarek AM, Balys RL, Jie S, Xu Y, Hier MP, Black MJ, Di Falco MR, LaBoissiere S, Alaoui-Jamali MA: Serum proteomic approach for the identification of serum biomarkers contributed by oral squamous cell carcinoma and host tissue microenvironment. J Proteome Res 2009, 8:2173-2185.

9. Hu HD, Ye F, Zhang DZ, Hu P, Ren H, Li SL: iTRAQ quantitative analysis of multidrug resistance mechanisms in human gastric cancer cells. $J$ Biomed Biotechnol 2010, 2010:571343.

10. Magharious M, D'Onofrio PM, Hollander A, Zhu P, Chen J, Koeberle PD: Quantitative iTRAQ analysis of retinal ganglion cell degeneration after optic nerve crush. J Proteome Res 2011, 10:3344-3362.

11. Matsumoto K, Satoh K, Maniwa T, Araki A, Maruyama R, Oda T: Noticeable decreased expression of tenascin- $\mathrm{X}$ in calcific aortic valves. Connect Tissue Res 2012, 50:460-468.

12. Qian WJ, Jacobs JM, Camp DG 2nd, Monroe ME, Moore RJ, Gritsenko MA, Calvano SE, Lowry SF, Xiao W, Moldawer LL, Davis RW, Tompkins RG, Smith RD: Comparative proteome analyses of human plasma following in vivo lipopolysaccharide administration using multidimensional separations coupled with tandem mass spectrometry. Proteomics 2005, 5:572-584.

13. Correale M, Brunetti ND, De Gennaro L, Di Biase M: Acute phase proteins in atherosclerosis (acute coronary syndrome). Cardiovasc Hematol Agents Med Chem 2008, 6:272-277.

14. Shirai R, Hirano F, Ohkura N, Ikeda K, Inoue S: Up-regulation of the expression of leucine-rich alpha(2)-glycoprotein in hepatocytes by the mediators of acute-phase response. Biochem Biophys Res Commun 2009, 382:776-779.

15. Kocsis J, Mészáros T, Madaras B, Tóth EK, Kamondi S, Gál P, Varga L, Prohászka Z, Füst G: High levels of acute phase proteins and soluble 70 $\mathrm{kDa}$ heat shock proteins are independent and additive risk factors for mortality in colorectal cancer. Cell Stress Chaperones 2011, 16:49-55.

16. Bode JG, Albrecht U, Häussinger D, Heinrich PC, Schaper F: Hepatic acute phase proteins-regulation by IL-6- and IL-1-type cytokines involving STAT3 and its crosstalk with NF-KB-dependent signaling. Eur J Cell Biol 2012, 91:496-505.

17. Ananian P, Hardwigsen J, Bernard D, Le Treut YP: Serum acute-phase protein level as indicator for liver failure after liver resection. Hepatogastroenterology 2005, 52:857-861.

18. Castell JV, Gómez-Lechón MJ, David M, Andus T, Geiger T, Trullenque R, Fabra $R$, Heinrich PC: Interleukin-6 is the major regulator of acute phase protein synthesis in adult human hepatocytes. FEBS Lett 1989, 242:237-239.

19. Ceron JJ, Eckersall PD, Martýnez-Subiela S: Acute phase proteins in dogs and cats: current knowledge and future perspectives. Vet Clin Pathol 2005, 34:85-99.

20. Saigo K, Yoshida A, Ryo R, Yamaguchi N, Leung LL: Histidine-rich glycoprotein as a negative acute phase reactant. Am J Hematol 1990, 34:149-150.

21. Daveau M, Rouet P, Scotte M, Faye L, Hiron M, Lebreton JP, Salier JP: Human inter-alpha-inhibitor family in inflammation: simultaneous synthesis of positive and negative acute-phase proteins. Biochem J 1993, 292:485-492.

22. Haas MJ, Mooradian AD: Regulation of high-density lipoprotein by inflammatory cytokines: establishing links between immune dysfunction and cardiovascular disease. Diabetes Metab Res Rev 2010, 26:90-99.

23. Didangelos A, Yin X, Mandal K, Saje A, Smith A, Xu Q, Jahangiri M, Mayr M: Extracellular matrix composition and remodeling in human abdominal aortic aneurysms: a proteomics approach. Mol Cell Proteomics 2011, 10:M111.008128.

24. Acosta-Martin AE, Panchaud A, Chwastyniak M, Dupont A, Juthier F, Gautier C, Jude B, Amouyel P, Goodlett DR, Pinet F: Quantitative mass spectrometry analysis using PACIFIC for the identification of plasma diagnostic biomarkers for abdominal aortic aneurysm. PLoS One 2011, 6:e28698.

25. Yin H, Gao L, Shen B, Chao L, Chao J: Kallistatin inhibits vascular inflammation by antagonizing tumor necrosis factor-alpha-induced nuclear factor kappaB activation. Hypertension 2010, 56:260-267. 
26. Gao L, Yin H, S Smith R Jr, Chao L, Chao J: Role of kallistatin in prevention of cardiac remodeling after chronic myocardial infarction. Lab Invest 2008, 88:1157-1166.

27. Rehman AA, Ahsan H, Khan FH: Alpha-2-macroglobulin: a physiological guardian. J Cell Physiol 2013, 228:1665-1675.

28. Rathinavel A, Dhandapany PS, Annapoorani P, Ramasamy S, Selvam GS: Cardiac isoform of alpha-2 macroglobulin as a novel diagnostic marker for cardiac diseases. Eur J Cardiovasc Prev Rehabil 2005, 12:601-603.

29. Sangiorgi G, D'Averio R, Mauriello A, Bondio M, Pontillo M, Castelvecchio S, Trimarchi S, Tolva V, Nano G, Rampoldi V, Spagnoli LG, Inglese L: Plasma levels of metalloproteinases- 3 and -9 as markers of successful abdominal aortic aneurysm exclusion after endovascular graft treatment. Circulation 2001, 104(Suppl I):I288-I295.

30. Matsumoto K, Maniwa T, Tanaka T, Satoh K, Okunishi H, Oda T: Proteomic analysis of calcified abdominal and thoracic aortic aneurysms. Int J Mol Med 2012, 30:417-429.

31. Matsumoto K: Phosphorylation of extracellular matrix tenascin- $X$ detected by differential mass tagging followed by NanoLC-MALDI-TOF/ TOF-MS/MS using ProteinPilot software. Connect Tissue Res 2012, 53:106-116

32. Shilov IV, Seymour SL, Patel AA, Loboda A, Tang WH, Keating SP, Hunter CL, Nuwaysir $L M$, Schaeffer DA: The paragon algorithm, a next generation search engine that uses sequence temperature values and feature probabilities to identify peptides from tandem mass spectra. Mol Cell Proteomics 2007, 6:1638-1655.

33. Nakamura Y, Takayama N, Minamitani T, Ikuta T, Ariga H, Matsumoto K Primary structure, genomic organization and expression of the major secretory protein of murine epididymis, ME1. Gene 2000, 251:55-62.

doi:10.1186/1477-5956-11-27

Cite this article as: Satoh et al:: Proteomic profiling for the identification of serum diagnostic biomarkers for abdominal and thoracic aortic aneurysms. Proteome Science 2013 11:27.

\section{Submit your next manuscript to BioMed Central and take full advantage of:}

- Convenient online submission

- Thorough peer review

- No space constraints or color figure charges

- Immediate publication on acceptance

- Inclusion in PubMed, CAS, Scopus and Google Scholar

- Research which is freely available for redistribution 